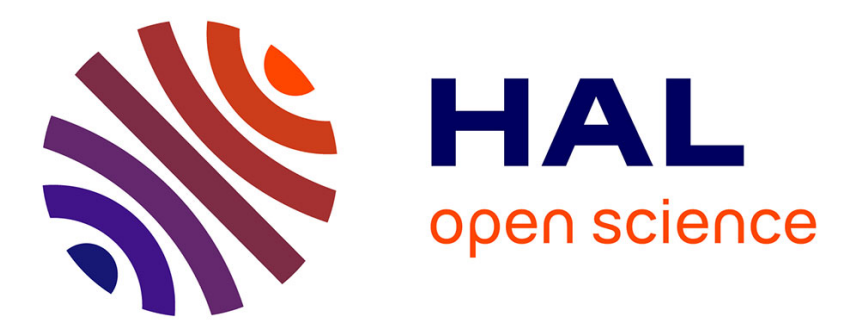

\title{
Modelling a Microscope as Low Dimensional Subspace of Operators
}

\author{
Valentin Debarnot, Paul Escande, Thomas Mangeat, Pierre Weiss
}

\section{To cite this version:}

Valentin Debarnot, Paul Escande, Thomas Mangeat, Pierre Weiss. Modelling a Microscope as Low

Dimensional Subspace of Operators. EUSIPCO 2020, Jan 2020, Amsterdam, France. hal-03092840

\section{HAL Id: hal-03092840 \\ https://hal.science/hal-03092840}

Submitted on 3 Jan 2021

HAL is a multi-disciplinary open access archive for the deposit and dissemination of scientific research documents, whether they are published or not. The documents may come from teaching and research institutions in France or abroad, or from public or private research centers.
L'archive ouverte pluridisciplinaire HAL, est destinée au dépôt et à la diffusion de documents scientifiques de niveau recherche, publiés ou non, émanant des établissements d'enseignement et de recherche français ou étrangers, des laboratoires publics ou privés. 


\section{Modelling a Microscope as Low Dimensional Subspace of Operators}

\author{
Valentin Debarnot \\ ITAV, USR 3505 \\ Université de Toulouse \\ Toulouse, France \\ valentindebarnot@gmail.com
}

\author{
Paul Escande \\ CNRS, IMM, Marseille \\ CNRS \\ Marseille, France \\ paulescande@gmail.com
}

\author{
Thomas Mangeat \\ CBI, Toulouse \\ CNRS
}

Toulouse, France

mangeatthomas@gmail.com pierrearmandweiss@gmail.com

\begin{abstract}
We propose a novel approach to calibrate a microscope. Instead of seeking a single linear integral operator (e.g. a convolution with a point spread function) that describes its action, we propose to describe it as a low-dimensional linear subspace of operators. By doing so, we are able to capture its variations with respect to multiple factors such as changes of temperatures and refraction indexes, tilts of optical elements or different states of spatial light modulator. While richer than usual, this description however suffers from a serious limitation: it cannot be used directly to solve the typical inverse problems arising in computational imaging. As a second contribution, we therefore design an original algorithm to identify the operator from the image of a few isolated spikes. This can be achieved experimentally by adding fluorescent micro-beads around the sample. We demonstrate the potential of the approach on a challenging deblurring problem.

Important note: this paper is an abridged version of a preprint [3] by the same authors, submitted for a journal publication.

Index Terms-fluorescence microscopy, PSF, calibration, product-convolution operators, blind deblurring
\end{abstract}

\section{INTRODUCTION}

The most common approach to calibrate a microscope consists in imaging fluorescent micro-beads and estimating a single point spread function (PSF) from this indirect information. This approach is based on the assumption that the optical system is space invariant and that the image formation model can be described by the mathematical model

$$
g=S(h \star f),
$$

where $g \in \mathbb{R}^{N}$ is the digital image, $f \in L^{2}\left(\mathbb{R}^{d}\right)$ is the ideal image, $h \in L^{2}\left(\mathbb{R}^{d}\right)$ is the convolution kernel or PSF and $S: L^{2}\left(\mathbb{R}^{d}\right) \rightarrow \mathbb{R}^{N}$ is a sampling operator. Despite being widespread, this model however suffers from two important limitations:

1) While space invariant systems are often accurate at a local scale, they are far from reflecting the complexity of microscope responses for larger fields of views [10], [9]. An example illustrating significant space variations of the point spread function on a wide-field microscope is shown on Fig. 1.

2) The imaging conditions (temperature, wavelength, tilts of optical elements,...) often change between the calibration step and the imaging of the sample, leading to

GDR ISIS, ANR OMS. model mismatches. These can have dramatic effects for computational tasks such as image deblurring or single molecule localization.

The aim of this paper is twofold:

- Provide a novel calibration approach which simultaneously solves the two above flaws, by learning a low dimensional model of space varying microscope responses. As an output, this algorithm provides a whole set of operators $\widehat{\mathcal{H}}$ which describe the possible transfer functions of the microscope.

- As such, this approach provides a fine metrological tool for opticians but cannot be directly used to solve inverse problems. When facing an image reconstruction problem, most existing approaches indeed require the knowledge of a single operator and not of a whole family. Our second contribution is to propose an algorithm that identifies an element of this subspace from an image containing a few separated spikes. This can be seen as a blind inverse problem solver: once the operator has been identified, it is possible to use standard solvers to actually reconstruct the image.

\section{LEARNING A LOW DIMENSIONAL SET OF OPERATORS}

For the first contribution, we need to turn to a more general framework than (1). We turn to the discrete setting with periodic boundary conditions for simplicity. We model the blurring operator $H: \mathbb{R}^{N} \rightarrow \mathbb{R}^{N}$ as a linear integral operator of the form

$$
H f(x)=\sum_{y \in \Omega} K(x, y) f(y),
$$

where $K$ is the kernel of the operator and $\Omega$ the pixels set. The function $L(x, y)=K(x+y, y)$ is called space varying impulse response. The point spread function (or impulse response) of the system at a location $y \in \mathbb{R}^{d}$ is defined by $L(\cdot, y)$. The forward observation model then becomes $y=H f+\eta$, for some noise $\eta \in \mathbb{R}^{N}$.

\section{A. Product-convolution}

Throughout this paper, we will assume that: 
- the impulse responses are well approximated by their projection on a low dimensional basis $\left(u_{i}\right)_{1 \leq i \leq I}$, i.e. for all $y \in \Omega$ :

$$
L(\cdot, y) \simeq \sum_{i=1}^{I}\left\langle L(\cdot, y), u_{i}\right\rangle u_{i}(\cdot)
$$

- the impulse responses vary slowly in space. This can be formalized by assuming that the function $y \mapsto L(x, y)$ (or that the mappings $y \mapsto\left\langle L(\cdot, y), u_{i}\right\rangle$ ) are smooth functions.

These two assumptions are crucial for this work. They allow to capture most of the effects of the couple (optical system, cover-slip). Let us mention however that they discard sharp transitions that may happen due to rapidly varying refractive indexes within a sample, or complex phenomena related to different dipole orientations.

Under these assumptions, we showed in [5] that the operator could be well approximated by a product-convolution expansion of the form:

$$
H f \simeq \sum_{i=1}^{I} \sum_{j=1}^{J} \gamma_{i, j} u_{i} \star\left(v_{j} \cdot f\right)
$$

where $\left(v_{j}\right)_{1 \leq j \leq J}$ are a set of multipliers and $\gamma_{i, j}$ a set of coefficients. Intuitively, the multipliers $v_{j}$ capture the space variations of the PSFs. In its simplest form, we could set $v_{j}=0$ for $j \neq i$ and $v_{i}(y)=\left\langle L(\cdot, y), v_{i}\right\rangle$. In that case, the equation (4) would boil down to the decomposition (3). The decomposition (4) has two significant assets:

- it can be implemented efficiently on a computer as a sum of $I$ convolutions. In practice, we observed that values of $I$ ranging between 3 and 7 depending on the SNR of the input images were enough to capture nearly all the PSF information.

- for a single operator, the bases $\left(u_{i}\right)$ and $\left(v_{j}\right)$ can be estimated efficiently by imaging sets of fluorescents beads as will be seen later [6], [8], [3].

\section{B. The learning procedure}

Let us mention that the mathematical foundations of the proposed approach have been established recently in [4]. However its specialization to the microscopy problem at hand raises many practical issues that will be discussed below.

For a given acquisition, a microscope can be described by a discrete linear operator $H: \mathbb{R}^{N} \rightarrow \mathbb{R}^{N}$. When varying the imaging conditions such as the temperature (which creates changes of refractive indexes), some focal screws or spatial light modulators, we actually obtain a complete family $\mathcal{H}$ of operators. The objective of this section is to estimate a structured set $\widehat{\mathcal{H}}$ that approximates $\mathcal{H}$ well.

Based on the previous discussion, we propose to describe the family $\widehat{\mathcal{H}}$ as a low-dimensional subspace of productconvolution operators. This means that we are looking for two families $\left(u_{i}\right)_{1 \leq i \leq I}$ and $\left(v_{j}\right)_{1 \leq j \leq J}$ such that for any $H \in \mathcal{H}$ and $f \in \mathbb{R}^{N}$, we have

$$
H f \simeq \sum_{i=1}^{I} \sum_{j=1}^{K} \gamma_{i, j} u_{i} \star\left(v_{j} \cdot f\right)
$$

for some matrix $\Gamma=\left(\gamma_{i, j}\right)$.

a) Experimental setup: To this end we propose to image small fluorescent microbeads under multiple conditions, which yields a sequence $\left(g_{1}, \ldots, g_{K}\right) \in \mathbb{R}^{N \times K}$ of images. Each of these images can be seen as the result of applying an operator $H_{k} \in \mathcal{H}$ to an unknown sparse measure.

In our experiments, we automatized this stage by using a motorized stage and a thermostatic chamber, allowing to vary the $x, y, z$ position of the sample, change the temperature and vary the position of a spatial light modulator (used for an automatic blur correction) automatically. The experiment of this paper will be performed with $K=378$ images containing between 100 and 200 micro-beads each.

b) Extraction of PSF patches: The second step consists in extracting a set of good quality PSF patches with a subpixel acccuracy of all the images in this sequence. This problem can sometimes be challenging due to i) low signal to noise ratio, ii) PSF overlapping and iii) clustering of micro-beads.

Ideally we would like each patch to contain a single PSF isolated from the rest and centered within the patch. The steps we propose are based on standard image processing tools: i) detection of potential PSFs by applying a Laplacian of Gaussian filter followed by a thresholding, ii) selection of patches containing a single maximum, iii) centering of the remaining patches by finding the best fit with a pyramid of Gaussian iv) discarding the likely outliers by performing specific $z$-tests among the patch population and v) resampling on a shifted grid using bi-cubic splines. We refer the interested reader to [3] for more details. As an output of this step, we have collected a (large) series of reliable PSF patches $\left(p_{1}, \ldots, p_{L}\right)$. The experiment of this paper will be performed with $L \simeq 23000$ detected patches.

c) Background removal: A critical step to correctly assess the basis $\left(u_{i}\right)$ is to remove the autofluorescence background from each PSF patch. As can be seen on Fig. ??, there are significant variations of intensity within a single image, due to a non homogeneous illumination and non uniform autofluorescence. It is necessary to correct for this effect. In this paper, we propose to fit a low degree polynomial to the patch boundary. The idea is that the boundary should not contain much energy from the PSF since they are decaying rapidly in space. As an output of this step, we obtain, a set of corrected patches $\left(\bar{p}_{1}, \ldots, \bar{p}_{L}\right)$.

d) The PSF basis $\left(u_{i}\right)$ : In this work we simply define the family $\left(u_{i}\right)_{1 \leq i \leq I}$ as the most significant elements of a principal component analysis applied to the sequence of patches $\left(\bar{p}_{1}, \ldots, \bar{p}_{L}\right)$. Notice that the singular values of the decomposition allow to choose the number of kept components 
$I$ in a principled way. In practice, this value varies between 3 and 7 . All the PSF patches $\bar{p}_{l}$ satisfy:

$$
\bar{p}_{l} \simeq \sum_{i=1}^{I}\left\langle\bar{p}_{l}, u_{i}\right\rangle u_{i} .
$$

e) The coefficient variations family $\left(v_{j}\right)$ : At this stage, for each micro-bead image $g_{k}$, we have access to a set of clean PSF patches $\left(\bar{p}_{l_{q}}\right)_{1 \leq q \leq Q_{k}}$, where $Q_{k} \in \mathbb{N}$ is the number of clean patches on the $k$-th im age, together with the positions $\left(x_{l_{q}}\right)_{1 \leq q \leq Q_{k}}$ of the PSFs within the image. In order to estimate the space variations of the PSFs, we propose to use a thin plate spline interpolation of the projection coefficients $\left\langle\bar{p}_{l_{q}}, u_{i}\right\rangle$ by solving:

$$
w_{k, i}=\underset{w \in \mathbb{R}^{N}}{\operatorname{argmin}} \frac{1}{2} \sum_{q=1}^{Q_{k}}\left(\left\langle\bar{p}_{l_{q}}, u_{i}\right\rangle-w\left(x_{l_{q}}\right)\right)^{2}+\frac{\lambda}{2}\|\Delta w\|_{2}^{2},
$$

where $\lambda>0$ is a regularization parameter that balances the smoothness of the map $w_{k, i}$ against the closeness of fit.

We perform this for all indexes $1 \leq i \leq I$ and images $1 \leq k \leq K$, leading to a set of $I K$ interpolation maps. We then propose to define the family $\left(v_{j}\right)$ by keeping the most significant principal components of the family $\left(w_{k, i}\right)$. Since the images $w_{k, i}$ are typically large, we resort to randomized singular value decompositions [7]. In practice, this leads to values of $J$ ranging from 4 to 10 , to capture the space variations precisely.

f) Restricting the family: At this stage, we learned a structured subspace of operators $\widehat{\mathcal{H}}$ of size $I J$, where the product $I J$ ranges in $\{12, \ldots, 70\}$ for a wide-field microscope producing large $2300 \times 2300$ images. Any element $H \in \widehat{\mathcal{H}}$ possesses the product-convolution structure (5). While expressing the list of all possible states of a microscope with a few dozen parameters is already impressive per se, it is possible to further reduce the family of admissible operators: not any matrix $\Gamma \in \mathbb{R}^{I \times J}$ leads to a decent operator. For instance, the operators should preserve the positivity of images, and the coefficients $\gamma_{i, j}$ are expected to decay with respect to $i$ and $j$ since these indexes are related to an ordering of singular values. In addition, the operator can be known only up to a positive multiplicative constant since the microbeads intensity is not controlled.

We therefore propose to restrict the family of admissible operators to the conical hull $\widehat{\mathcal{C}} \subsetneq \widehat{\mathcal{H}}$ of the observed operators. Letting $\left(\widehat{\Gamma}_{k}\right)$ denote the family of $I \times J$ matrices estimated from the different micro-beads images, we can define the final set of admissible operators as:

$$
\widehat{\mathcal{C}}:=\operatorname{cone}\left(H\left(\widehat{\Gamma}_{k}\right)\right)=\left\{\sum_{k=1}^{K} \lambda_{k} H\left(\widehat{\Gamma}_{k}\right), \lambda_{k} \geq 0\right\},
$$

where $H\left(\widehat{\Gamma}_{k}\right) f:=\sum_{i=1}^{I} \sum_{j=1}^{J} \hat{\gamma}_{i, j}^{k} u_{i} \star\left(v_{j} \cdot f\right)$. In the companion journal paper [3], we further propose to simplify this set by designing a conical peeling algorithm.

An estimated family of operators is displayed in Fig. 2. The top row corresponds to the family $\left(u_{i}\right)$ while the bottom row corresponds to the family $\left(v_{j}\right)$.

\section{BLIND BLUR IDENTIFICATION}

The proposed methodology departs significantly from the usual calibration procedures which yield a single operator that can be used directly for solving the subsequent inverse problems. Here, we need to infer the operator from the observed data, which is the field of blind inverse problems. While this is a hard and open problem in general, the learning procedure described previously significantly eases its resolution. Instead of looking for an arbitrary space varying blur operator, we just need to estimate $I \times J$ coefficients restricted to the low diameter conical hull $\widehat{\mathcal{C}}$.

Unfortunately, this is still an open problem in general despite recent theoretical progresses [1]. To further simplify the problem, we assume that it is possible to insert isolated fluorescent micro-beads around the sample, which then provide a few scattered impulse responses that can be used for the estimation.

\section{A. The experimental and mathematical framework}

We assume that the observed image $f$ reads as

$$
f=f_{0}+\sum_{p=1}^{P} \alpha_{p} \delta_{x_{p}}
$$

where $f_{0}$ is the sample we wish to image, $P$ is the number of scattered micro-beads (or more generally sub-diffraction limit point sources), and $\left(x_{p}\right)$ is a set of separated positions in $\mathbb{R}^{d}$.

Under this assumption, the observed image $g=g_{0}+g_{b}+\eta$ is the sum of a blurry signal $g_{0}=H\left(f_{0}\right)$, the image of a sparse measure $g_{b}=\sum_{p=1}^{P} \alpha_{p} K\left(\cdot, x_{p}\right)$ and the noise $\eta$. As mentioned previously, a simple thresholding of the Laplacian of Gaussian or a manual selection are enough to find the locations $x_{p}$. This in turn allows to extract a few patches $\omega_{p}$ centered at $x_{p}$ containing isolated and noisy point spread functions. Following the methodology described in the previous section, it is possible to remove the background (here the component $g_{0}$ ) from the patches to recover masked images $s_{p}=\left(H \delta_{x_{p}}+\eta\right) \mathbb{1}_{\omega_{p}}$ containing only the $p$-th noisy PSF. Notice that we implicitely assumed here that the PSFs were sufficiently far apart so that the contribution from one PSF does not interfere with the others.

\section{B. The identification algorithm}

Assuming that $H=H(\Gamma) \in \widehat{\mathcal{H}}$, a natural approach to identify $\Gamma$ from $f$ consists in solving the following bilinear inverse problem:

$$
\widehat{\Gamma}=\underset{\Gamma \in \mathbb{R}^{I \times J}, \alpha \in \mathbb{R}^{P}}{\operatorname{argmin}} \frac{1}{2} \sum_{p=1}^{P}\left\|\alpha_{p} H(\Gamma) \delta_{x_{p}}-s_{p}\right\|_{2}^{2} .
$$

In words, we wish to find an operator $H(\Gamma)$ that - when applied to Dirac masses at $x_{p}$ - reproduces the patches $s_{p}$ up to an unknown multiplicative constant $\alpha_{p}$.

Problem (10) is non convex and looks high-dimensional at first sight. However the specific product-convolution structure simplifies it significantly. Let

$$
U_{p}=\left[u_{1}\left(\cdot-x_{p}\right), \ldots, u_{I}\left(\cdot-x_{p}\right)\right] \in \mathbb{R}^{N \times I}
$$


denote the orthogonal basis describing the PSFs shifted by $x_{p}$. Since, by definition,

$$
H(\Gamma) \delta_{x_{p}}=\sum_{i=1}^{I} \sum_{j=1}^{J} \gamma_{i, j} v_{j}\left(x_{p}\right) u_{i}\left(\cdot-x_{p}\right)
$$

we get

$$
\begin{aligned}
& \widehat{\Gamma}=\underset{\Gamma \in \mathbb{R}^{I \times J}, \alpha \in \mathbb{R}^{P}}{\operatorname{argmin}} \frac{1}{2} \sum_{p=1}^{P}\left\|U_{p}^{*}\left(\alpha_{p} H(\Gamma) \delta_{x_{p}}-s_{p}\right)\right\|_{2}^{2} \\
& =\underset{\Gamma \in \mathbb{R}^{I \times J}, \alpha \in \mathbb{R}^{P}}{\operatorname{argmin}} \frac{1}{2} \sum_{p=1}^{P} \sum_{i=1}^{I}\left(\sum_{j=1}^{J} \alpha_{p} \gamma_{i, j} v_{j}\left(x_{p}\right)-s_{p, i}\right)_{2}^{2},
\end{aligned}
$$

with $s_{p, i}=\left\langle s_{p}, u_{i}\left(\cdot-x_{p}\right)\right\rangle$. If the amplitudes $\alpha_{p}$ were known, the previous problem would be a simple linear least squares. A necessary condition for identifiability of $\Gamma$ would be that the number of bead $P$ exceeds $J$, the number of space variation maps.

With the additional unknown $\alpha$, the problem is nonconvex, but can be lifted to a convex problem, following [1]. As mentioned earlier, the values $I, J$ and $P$ are typically low and this technique can therefore be applied without pain.

\section{Remark 1:}

- Notice that the sum over $p$ is outside the norm in (10). This is valid only if the Dirac masses are separated, in the sense that the energy of the PSFs different from $p$ can be neglected when restricted to $\omega_{p}$ (or at least that their contribution can be removed with the background removal algorithm). It would be possible to keep the sum inside the norm, but the numerical complexity would be much higher.

- As usual in bilinear inverse problems, there is an ambiguity between the amplitudes of the Dirac masses and the amplification by the operator $H(\Gamma)$ : we can multiply $\alpha$ by a nonzero factor, divide $\Gamma$ by the same factor and the energy in (10) is kept unchanged. To avoid this ambiguity, we can choose a reference matrix $\Gamma_{0} \in \mathbb{R}^{I \times J}$ and add a linear constraint $\left\langle\Gamma, \Gamma_{0}\right\rangle=1$.

- Solving the bilinear inverse problem (10) can be enough to recover the operator $H$. However, when the number $P$ of observed PSFs is small, it is possible to add safeguards by imposing $\Gamma$ to live on the conical hull cone $\left(\widehat{\Gamma}_{k}, 1 \leq\right.$ $k \leq K)$, since we know that the operator should live in $\widehat{\mathcal{C}}$, see (8). In that case, Problem (10) can be solved with the alternating projected gradient descent developed in [3].

\section{Sparse plus smooth deblurring}

As an output of the previous algorithm, we obtain a matrix $\widehat{\Gamma}$ as well as an estimation $\widehat{H}=H(\widehat{\Gamma})$ of the true underlying operator $H$. It is possible to use this estimate directly within a standard inverse problem solver to reconstruct the desired signal. We propose an original solver for sparse spikes deconvolution below:

$\inf _{f_{1} \in \mathbb{R}_{+}^{N}, f_{2} \in \mathbb{R}_{+}^{N}} \frac{1}{2}\left\|\widehat{H}\left(f_{1}+f_{2}\right)-f\right\|_{2}^{2}+\frac{\lambda_{1}}{2}\left\|\Delta u_{1}\right\|_{2}^{2}+\frac{\lambda_{2}}{2}\left\|u_{2}\right\|_{1}$.

The underlying idea is to find two components:

- $f_{1}$ corresponds to the autofluorescence background. It is assumed to be smooth and we therefore resort to an $H^{2}$ regularization with the Laplacian, which appears in thinplate splines smoothing [2].

- $f_{2}$ corresponds to the sparse measure, which is typically the object of interest for single molecule localization microscopy for instance.

A result of the proposed methodology is shown on Fig. 3. Notice that the centroids of the microbeads are all recovered despite significant space variations.

\section{CONCLUSiON}

We proposed a new methodology to calibrate a microscope together with a new algorithm to identify the blur from blurry images containing subdiffraction point sources. The proposed methodology provide very promising results on challenging large scale images of wide-field microscopy. This work opens many avenues. Of particular interest, let us mention that it allows to simulate a large number of realistic degradations and to train neural networks for reconstruction tasks.

\section{ACKNOWLEDGMENT}

This work was supported by the GDR ISIS project Filmosuremi, the ANR Optimization on Measures Spaces and the ANR-3IA Artificial and Natural Intelligence Toulouse Institute.

\section{REFERENCES}

[1] Ali Ahmed, Benjamin Recht, and Justin Romberg. Blind deconvolution using convex programming. IEEE Transactions on Information Theory, 60(3):1711-1732, 2013.

[2] Martin D Buhmann. Radial basis functions: theory and implementations, volume 12. Cambridge university press, 2003.

[3] Valentin Debarnot, Paul Escande, Thomas Mangeat, and Pierre Weiss. Learning low-dimensional models of microscopes. preprint, January 2020.

[4] Valentin Debarnot, Paul Escande, and Pierre Weiss. A scalable estimator of sets of integral operators. Inverse Problems, 35(10):105011, 2019.

[5] Paul Escande and Pierre Weiss. Approximation of integral operators using product-convolution expansions. Journal of Mathematical Imaging and Vision, 58(3):333-348, 2017.

[6] Marc Gentile, Frederic Courbin, and Georges Meylan. Interpolating point spread function anisotropy. Astronomy \& Astrophysics, 549:A1, 2013.

[7] Nathan Halko, Per-Gunnar Martinsson, and Joel A Tropp. Finding structure with randomness: Probabilistic algorithms for constructing approximate matrix decompositions. SIAM review, 53(2):217-288, 2011.

[8] Jérémie Bigot and Paul Escande and Pierre Weiss. Estimation of linear operators from scattered impulse responses. Applied and Computational Harmonic Analysis, 47(3):730 - 758, 2019.

[9] Alex von Diezmann, Maurice Y Lee, Matthew D Lew, and WE Moerner. Correcting field-dependent aberrations with nanoscale accuracy in three-dimensional single-molecule localization microscopy. Optica, 2(11):985-993, 2015.

[10] Guoan Zheng, Xiaoze Ou, Roarke Horstmeyer, and Changhuei Yang. Characterization of spatially varying aberrations for wide field-of-view microscopy. Optics express, 21(13):15131-15143, 2013. 


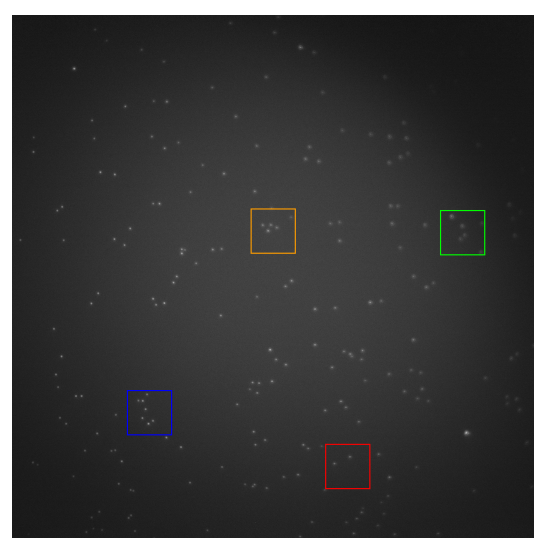

(a) $2304 \times 2304$ image
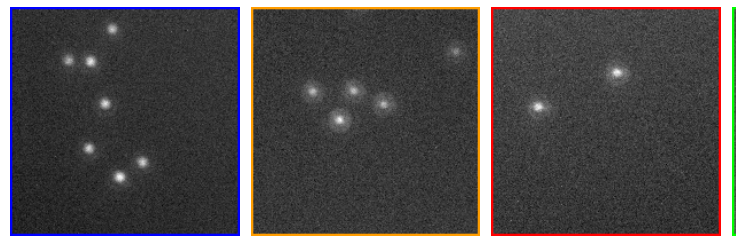

(b) Original image.
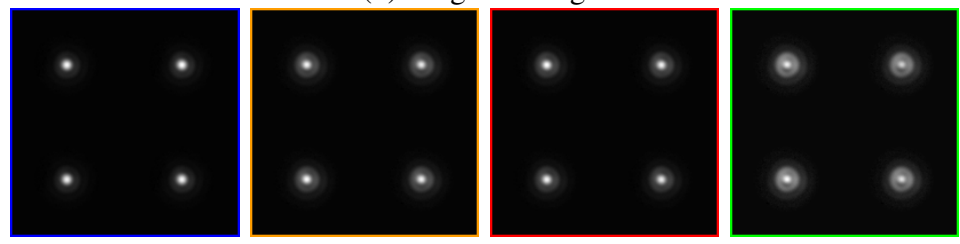

(c) Operator estimated from a family of 18 images.

Fig. 1: Left: micro-beads imaged with a wide field microscope. Right: estimated operator with the proposed methodology.
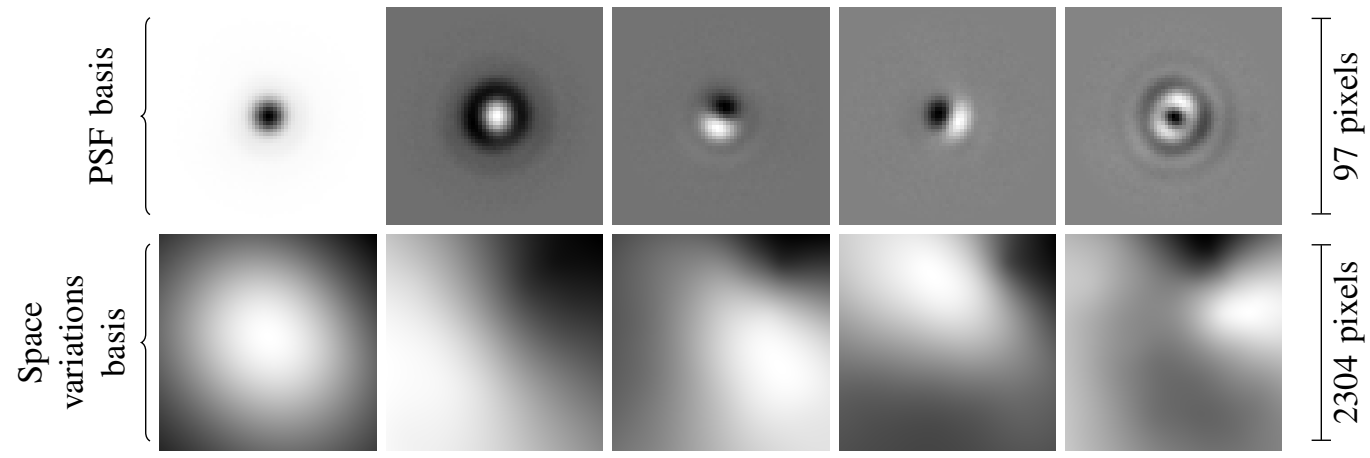

Fig. 2: Learning the PSF and space variations bases for a standard wide-field microscope.
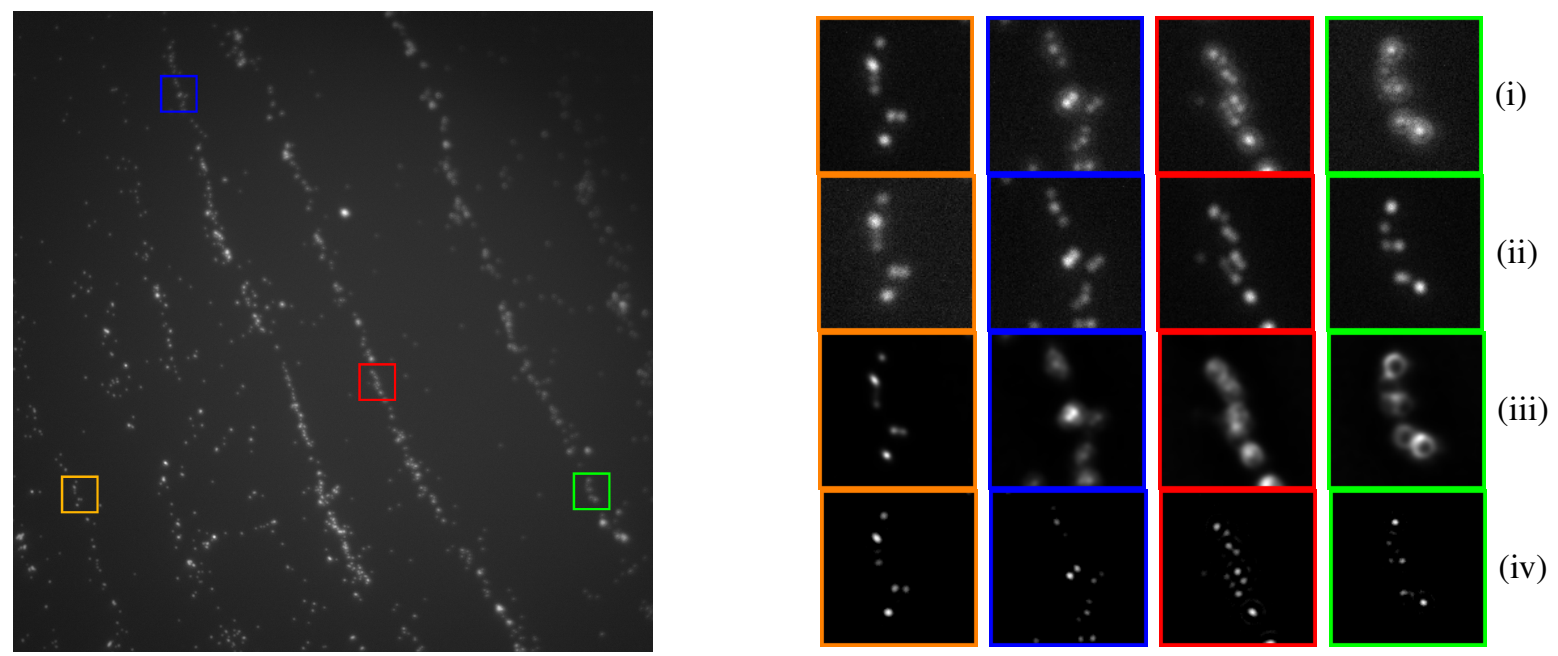

(a) Clusters of micro-beads imaged at 300nm from the focal plane.

(b) (i) original out of focus. (ii) original at focal plane. (iii) The contrasts have been stretched for a better visualization. deblurred with Huygens software. (iv) BSS deblurring.

Fig. 3: Blind deblurring of clustered microbeads. Observe how well the micro-beads centers are recovered despite significant space variations and unknown blurring operator. 